Table 1. Significant correlations with the OCI-R for: a) all SLE participants; b) the SLE-F group only (visit 1 minus visit 2).

\begin{tabular}{lcc}
\hline Variable & rs & p-value \\
\hline a)All SLE participants, $\mathrm{n}=39$ & & \\
Disease activity: BILAG global score & 0.408 & 0.01 \\
Quality of life: & & \\
$\quad$ LupusQoL - Physical & -0.495 & 0.001 \\
$\quad$ - Pain & -0.535 & $<0.001$ \\
$\quad$ - Planning & -0.586 & $<0.001$ \\
$\quad$ - Intimate & -0.342 & 0.03 \\
- Burden & -0.504 & 0.001 \\
$\quad$ - Emotion & -0.397 & 0.01 \\
$\quad$ - Fatigue & -0.471 & 0.002 \\
EQ5D: VAS & -0.418 & 0.01 \\
$\quad$ total & -0.359 & 0.03 \\
Fatigue measures (FSMC): & & \\
Cognitive & 0.521 & 0.001 \\
Motor & 0.448 & 0.004 \\
Depression measures: & & \\
MADRS & 0.467 & 0.003 \\
HADS - D & 0.545 & $<0.001$ \\
Anxiety measure: HADS-A & 0.375 & 0.02 \\
b)SLE-F group (v1-v2), $\mathbf{n = 1 1}$ & & \\
Inflammatory marker: MCP-1 & 0.771 & 0.006 \\
\hline
\end{tabular}

BILAG The British Isles Lupus Assessment Group index, LupusQoL Lupus quality of life, EQ5D European quality of life, VAS visual analogue scale, FSMC Fatigue scale for motor and cognitive function, MADRS Montgomery Asberg depression rating scale, HADS Hospital anxiety and depression scale, D-depression, A-anxiety score, MCP-1 monocyte chemoattractant protein-1

Genzyme Sanofi, GSK, and UCB, Consultant of: Eli Lilly, AstraZeneca, UCB, Iltoo, and Merck Serono, Speakers bureau: UCB

DOI: 10.1136/annrheumdis-2020-eular.528

\section{AB0403 VALIDATION OF 2019 EULAR/ACR CLASSIFICATION CRITERIA FOR SYSTEMIC LUPUS ERYTHEMATOSUS IN A SOUTH INDIAN COHORT}

M. Chalakkarayil Bhagavaldas ${ }^{1}$, H. B ${ }^{1}$, S. Balan ${ }^{1}{ }^{1}$ Amrita Institute of Medical Sciences, Kochi, India

Background: New 2019 EULAR/ACR classification criteria for Systemic Lupus Erythematosus (SLE) has been recently published ${ }^{1}$. Before its widespread use in research, this criterion needs to be externally validated in other cohorts. Validation studies for the new criteria were not yet done in the Indian population. Moreover, the new 2019 EULAR/ACR classification criteria for SLE has not been validated in juvenile SLE.

Objectives: To compare the real-world performance of 2019 EULAR/ACR classification criteria when applied to a known cohort of SLE cases, comprising both adult and juvenile SLE, in South Indian population.

Methods: We retrospectively reviewed the electronic medical record of 30,541 patients who visited the Rheumatology department of Amrita Institute of Medical Sciences, Kochi, a tertiary care centre in Kerala, from January 2014 to June 2019. 347 patients diagnosed with SLE by qualified and experienced rheumatologists were included in the study. 44 patients were later excluded as they were found to have an overlap syndrome. From the 30,541 patients, another 303 age-matched patients, who attended the rheumatology department on the first week of each month, from 2014 January to 2019 June, and diagnosed with autoimmune diseases other than SLE, were selected as controls. They were selected regardless of their specific clinical or immunologic manifestations. Patients were excluded if the diagnosis was uncertain. Each patient was evaluated to see if he or she satisfied the 1997, 2012, and 2019 classification criteria, respectively.

Results:

Conclusion: In our cohort, the new 2019 EULAR/ACR criteria attained better sensitivity, PPV, NPV and accuracy when compared to ACR 1997 and SLICC

Validation of the 2019 SLE classification criteria

\begin{tabular}{lcccccccccc}
\hline Criteria & \multicolumn{3}{c}{ Adult $(\mathrm{n}=196)$} & \multicolumn{3}{c}{ Juvenile $(\mathrm{n}=107)$} & \multicolumn{3}{c}{ Total $(\mathrm{n}=303)$} \\
\cline { 2 - 10 } & 1997 & 2012 & 2019 & 1997 & 2012 & 2019 & 1997 & 2012 & 2019 \\
\hline Sensitivity(\%) & 78.1 & 96.4 & 98.5 & 73.8 & 94.4 & 96.2 & 76.6 & 95.7 & 97.7 \\
Specificity(\%) & 92.9 & 86.2 & 93.4 & 100 & 96.3 & 98.1 & 95.4 & 89.8 & 95 \\
PPV $^{\#}(\%)$ & 91.6 & 87.5 & 93.7 & 100 & 96.2 & 98.1 & 94.3 & 90.3 & 95.2 \\
NPV $^{*}(\%)$ & 80.9 & 96 & 98.4 & 79.3 & 94.5 & 96.3 & 80.3 & 96.5 & 97.6 \\
Accuracy(\%) & 85.5 & 91.3 & 95.9 & 86.9 & 95.3 & 97.2 & 86 & 92.7 & 96.4 \\
\hline
\end{tabular}

\#PPV- Positive Predictive Value *NPV- Negative Predictive Value
2012 criteria, and had almost the same specificity as compared to SLICC 2012 and ACR 1997 criteria.

\section{References:}

[1] Aringer M, Costenbader K, Daikh D, et al. 2019 European League against Rheumatism/American College of rheumatology classification criteria for systemic lupus erythematosus. Ann Rheum Dis2019;78:1151-9

[2] Petri M, Orbai A-M, Alarcón GS, et al. Derivation and validation of the systemic lupus international collaborating clinics classification criteria for systemic lupus erythematosus. Arthritis Rheumatism 2012;64:2677-86

[3] Hochberg MC. Updating the American College of rheumatology revised criteria for the classification of systemic lupus erythematosus. Arthritis Rheum1997;40

Disclosure of Interests: None declared

DOI: 10.1136/annrheumdis-2020-eular.306

\section{AB0404 SARCOPENIA AND RELATED FACTORS AMONG PATIENTS WITH PRIMARY SJOGREN'S SYNDROME}

S. Colak ${ }^{1}$, E. Tekgoz ${ }^{1}$, S. Hayme ${ }^{2}$, I. Sonaeren ${ }^{3}$, M. Çınar ${ }^{1}$, S. Yilmaz ${ }^{1}$. ${ }^{1}$ University of Health Sciences Gulhane Medicine Faculty, Department of Internal Medicine, Division of Rheumatology, Ankara, Turkey; ${ }^{2}$ Ankara University Faculty of Medicine, Department of Biostatistics, Ankara, Turkey; ${ }^{3}$ Gulhane Training and Research Hospital, Department of Nutrition and Dietetics, Ankara, Turkey

Background: Sarcopenia is the progressive and generalized loss of muscle mass, strength and function especially among elderly population. Inflammation may lead to sarcopenia regardless of age.

Objectives: To evaluate the frequency of sarcopenia and related factors in patients with primary Sjogren's syndrome (SS).

Methods: A total of 44 female patients with SS and 44 age matched female healthy controls were included in this cross-sectional study. Sarcopenia was evaluated by hand grip test, skeletal muscle index (SMI) and 6 meters gait speed (GS) test. According to recommendations of European Working Group on Sarcopenia in Older People (EWGSOP2) 2018, sarcopenia is defined as decrease in results of both hand grip test and SMI, whereas, probable sarcopenia is defined as only decrease in results of hand grip test. Mini Nutritional Assessment Short Form (MNA-SF) was used for evaluating nutritional statement. EULAR SS patient reported index (ESSPRI) and EULAR SS disease activity index (ESSDAI) used for evaluating disease activity. Patient global assessment (PGA) was assessed with visual analogue scale (VAS $0-10 \mathrm{~cm}$ ). Patients with arthritis in dominant hand and/ or ankle joints were excluded from the study.

Results: The mean age of participants was $55.3 \pm 10.4$ years. Eleven patients $(25.0 \%)$ had probable sarcopenia in SS group and $2(4.5 \%)$ in control group $(p=0.007)$. Compared with healthy controls, SS patients had lower results of hand grip and 6 meters GS tests ( $p=0.005$ and $p<0.001$, respectively). According to Mini Nutritional Assessment Short Form (MNA-SF), patients with probable sarcopenia had higher risk for malnutrition compared with patients with no sarcopenia $(p=0.043)$. Patients with probable sarcopenia had higher scores of ESSPRI pain domain and patient visual analogue scale for global disease activity compared with patients with no sarcopenia $(p=0.044$ and $p=0.036$ respectively) (Table 1 ). In multivariate regression analysis ESSPRI pain was associated with hand grip strength $\left(p=0.016, R^{2}=0.13\right)$ and MNA was associated with SMI ( $p=0.005)$ (Table 2).

Table 1. Factors associated with probable sarcopenia in Sjogren's syndrome

\begin{tabular}{lccc}
\hline Sjogren's syndrome group & $\begin{array}{c}\text { No sarcopenia } \\
(\mathbf{n}=\mathbf{3 3})\end{array}$ & $\begin{array}{c}\text { Probable Sarcopenia } \\
(\mathbf{n}=\mathbf{1 1})\end{array}$ & p-value \\
\hline ESSPRI pain* & $5(0-10)$ & $7(3-10)$ & $\mathbf{0 . 0 4 4 ^ { \mathbf { b } }}$ \\
VAS patient* & $4(0-10)$ & $6(0-10)$ & $\mathbf{0 . 0 3 6}^{\mathbf{b}}$ \\
MNA SF, n (\%) & $32(97)$ & $8(72.7)$ & $\mathbf{0 . 0 4 3}^{\mathrm{C}}$ \\
Normal nutritional status (12-14) & $1(3)$ & $3(27.3)$ & \\
Risk of malnutrition (8-11) & & \\
\hline
\end{tabular}

ESSPRI: EULAR Sjogren's Syndrome Patient Reported Index, VAS: Visual Analogue Scale, MNA SF: Mini Nutritional Assessment Short Form. *Variables given as median (minimum-maximum) ${ }^{\mathrm{a}}$ Independent Samples Student $t$ test, ${ }^{\mathrm{b}}$ Mann-whitney $\mathrm{U},{ }^{\mathrm{C}}$ Fisher's Exact test. 
Table 2. Multivariate analysis for hand grip strength and SMI in patients with Sjogren's syndrome

\begin{tabular}{|c|c|c|c|c|}
\hline $\begin{array}{l}\text { Unstandardized } \\
\text { Coefficients }\end{array}$ & & $\begin{array}{l}95 \% \text { Confidence } \\
\text { Interval for B }\end{array}$ & & $\begin{array}{l}\text { Standardized } p \text {-value } \\
\text { Coefficients }\end{array}$ \\
\hline B & $\begin{array}{l}\text { Std. } \\
\text { Error }\end{array}$ & Lower & Upper & Beta \\
\hline
\end{tabular}

Hand grip

strength

Constant

ESSPRI pain

SMI

Constant

MNA

24,492

$-0,707$

1,695
0,282

21,071
$-1,276$

27,914

27,914
$-0,139$

$-0,361$

$<0.001$

5,419

1,705

1,978

8,860

$-0,139$

$-$

0.003

ESSPRI: EULAR Sjogren's Syndrome Patient Reported Index, SMI: Skeletal Muscle Index, MNA: Mini Nutritional Assessment

Conclusion: Risk of sarcopenia is increased in patients with SS. In the current study, it is shown that pain is related with sarcopenia. ESPPRI pain is a sign of continuing chronic inflammation in patients with SS. Malnutrition, which can indirectly related with SS, may also contribute to this process. Excessive pain may lead to decrease daily activities and nutritional status of patients with SS. Evaluating pain and patient's global disease activity may help physicians to find out patients with increased risk for sarcopenia. Controlling disease activity and pain and preventing malnutrition may reduce the risk for development of sarcopenia.

Acknowledgments : None to declare

Disclosure of Interests: : None declared

DOI: 10.1136/annrheumdis-2020-eular.4918

\section{AB0405 PREMENOPAUSAL FEMALE SYSTEMIC LUPUS ERYTHEMATOSUS PATIENTS IS RELATED TO HYPERURICEMIA AND PROTEINURIA}

H. Liu ${ }^{1}$, J. Z. Lin ${ }^{2}$, X. Cai ${ }^{3}$, J. D. MA ${ }^{2}$, Y. Mo ${ }^{2}$, M. Xie ${ }^{1}$, L. Dai ${ }^{2}{ }^{1}$ Panyu Central Hospital, Department of Rheumatology, Guangzhou, China; ${ }^{2}$ Sun Yat-Sen Memorial Hospital, Sun Yat-sen University, Department of Rheumatology, Guangzhou, China; ${ }^{3}$ Guangzhou First People's Hospital, The Second Affiliated Hospital of South China University of Technology, Department of Rheumatology, Guangzhou, China

Background: 1. Hyperuricemia is often associated with abnormal lipid metabolism. We reported premenopausal female systemic lupus erythematosus (SLE) patients had elevated blood UA levels ${ }^{[1]}$. Do these patients also have hyperlipidemia?

2. Estrogen has certain effect on blood lipid metabolism, whether the blood lipid levels of premenopausal female SLE patients who have the background of hyperestrogen function are affected by estrogen and its receptors?

Objectives: To investigate the relationships between blood lipids and serum UA level, estrogen receptors (ERs) as well as ER antibodies in premenopausal female SLE patients.

Methods: 123 premenopausal female SLE patients (SLE group) were divided into normal $\mathrm{CH}$ group $(\mathrm{n}=93)$ and high $\mathrm{CH}$ group $(\mathrm{n}=30, \mathrm{CH}>5.17 \mathrm{mmol} / \mathrm{l})$, and 40 healthy premenopausal females served as the control group. The blood lipid levels of the SLE group and the control group were compared, and the blood levels of lipid, UA, estrogen, ERs and ER antibodies were compared between the two SLE subgroups. Linear regression was used to analyze the influencing factors of blood $\mathrm{CH}$.

Results:

1. In SLE group, the blood level of TG was significantly higher than that of the control group $(1.67 \pm 1.10$ vs. $0.87 \pm 0.47, P<0.001)$, while the levels of blood $\mathrm{CH}$, LDL, HDL were comparable to the control group (all with $\mathrm{P}>0.05$ ).

2. The mean blood $\mathrm{CH}$ level of the SLE patients with hyperuricemia was $5.57 \pm$ $2.44 \mathrm{mmol} / \mathrm{l}$, which was significantly higher than that of patients with normal UA level $(3.98 \pm 1.30 \mathrm{mmol} / \mathrm{I}, \mathrm{P}<0.001)$.

3. The serum UA, CRE, CH, TG, LDL, and 24-hour urinary protein quantification (24h UPRO) in the high CH SLE subgroup were significantly higher than those in the normal $\mathrm{CH}$ SLE subgroup (all with $\mathrm{P}<0.05$ ). There were no significant differences in serum estrogen, ERs and ER antibodies between the two subgroups, Table 1.

4. Linear regression showed that serum UA level and $24 \mathrm{~h}$ UPRO were the dangerous effects of elevated blood $\mathrm{CH}$ in the premenopausal female SLE patients, Table 2

Conclusion: Compared with healthy female of the same age range, the premenopausal female SLE patients are more likely to have abnormal lipid metabolism, which is related to kidney damage and abnormal UA metabolism.

\section{References:}

[1] Haijun Liu, Xiaoyan Cai, Lie Dai, et al. Elevated uric acid levels in premenopausal female systemic lupus erythematosus patients: association with potential or existing renal damage. Eur J Inflamm,2018,16:1-6.

Table 1. Some clinical indicators, estrogen, ERs and ER antibodies in the premenopausal female SLE patients

\begin{tabular}{lccc}
\hline & $\begin{array}{c}\text { High } \mathbf{C H} \text { group } \\
(\mathbf{n}=\mathbf{3 0})\end{array}$ & $\begin{array}{c}\text { Normal } \mathbf{C H} \text { group } \\
(\mathbf{n}=\mathbf{9 3})\end{array}$ & $\mathbf{P}$ \\
\hline Age(years) & $31.40 \pm 7.40$ & $28.12 \pm 7.23$ & 0.039 \\
UA $(\mu \mathrm{mol} / \mathrm{l})$ & $433.40 \pm 192.90$ & $310.10 \pm 129.57$ & 0.002 \\
$\mathrm{CRE}(\mu \mathrm{mol} / \mathrm{l})$ & $112.07 \pm 87.09$ & $72.81 \pm 91.96$ & 0.039 \\
$\mathrm{CH}(\mathrm{mmol} / \mathrm{l})$ & $7.07 \pm 2.05$ & $3.72 \pm 0.93$ & $<0.001$ \\
$\mathrm{TG}(\mathrm{mmol} / \mathrm{l})$ & $2.27 \pm 1.57$ & $1.48 \pm 0.84$ & 0.013 \\
$\mathrm{LDL}(\mathrm{mmol} / \mathrm{l})$ & $4.48 \pm 1.46$ & $2.29 \pm 0.67$ & $<0.001$ \\
$\mathrm{HDL}(\mathrm{mmol} / \mathrm{l})$ & $1.02 \pm 0.72$ & $1.97 \pm 0.43$ & 0.721 \\
Estrogen(pg/ml) & $73.53 \pm 47.74$ & $69.42 \pm 55.48$ & 0.717 \\
ERa(pg/ml) & $45.94 \pm 31.06$ & $43.34 \pm 31.05$ & 0.684 \\
ER $(\mathrm{pg} / \mathrm{ml})$ & $16.65 \pm 28.67$ & $13.70 \pm 25.09$ & 0.589 \\
Anti-ERa(ng/ml) & $59.75 \pm 33.87$ & $55.16 \pm 31.34$ & 0.496 \\
Anti-ER $\beta(\mathrm{ng} / \mathrm{ml})$ & $89.73 \pm 49.03$ & $71.73 \pm 41.74$ & 0.052 \\
$24 \mathrm{~h}$ UPRO(g/24h) & $2.82 \pm 3.82$ & $0.39 \pm 0.56$ & 0.002 \\
Positive UBLD(\%) & $20(66.67)$ & $31(33.33)$ & 0.01 \\
SLEDAl & $13.18 \pm 4.23$ & $7.39 \pm 6.18$ & 0.025 \\
& & &
\end{tabular}

ER, estrogen receptor; 24h UPRO, 24-hour urinary protein quantification; UBLD, urinary blood.

Table 2. Relationships between blood $\mathrm{CH}$ level and clinical indicators in in the premenopausal female SLE patients

\begin{tabular}{lccccccc}
\hline Variable & $\begin{array}{c}\text { Unstandardized } \\
\text { Coefficients }\end{array}$ & & $\begin{array}{c}\text { Standardized } \\
\text { Coefficients }\end{array}$ & t & P & 95\% Cl \\
& B & SEM & & & & & \\
\hline 24h UPRO & 0.353 & 0.072 & 0.400 & 4.932 & $<0.001$ & $0.211 \sim 0.495$ \\
UA & 0.003 & 0.001 & 0.249 & 3.058 & 0.003 & $0.001 \sim 0.005$ \\
Constant & 3.146 & 0.359 & & 8.754 & $<0.001$ & $2.434 \sim 3.857$
\end{tabular}

UA, uric acid; 24h UPRO, 24-hour urinary protein quantification.

Disclosure of Interests: : None declared

DOI: 10.1136/annrheumdis-2020-eular.2696

\begin{tabular}{|l|l|}
\hline AB0406 & HUMAN PAPILLOMA VIRUS (HPV) VACCINATION \\
SAFETY IN SYSTEMIC LUPUS ERYTHEMATOSUS \\
COHORT - PORTUGUESE UNIVERSITY HOSPITAL \\
SINGLE-CENTER COHORT STUDY
\end{tabular}

J. Sousa Morais ${ }^{1}$, D. G. Oliveira ${ }^{2}$, R. Faria ${ }^{3,4}$, A. Almeida $^{3}$, M. Brandão $0^{3,4}$, A. Marinho ${ }^{3,4}$, I. Almeida ${ }^{3,4}$, F. Farinha ${ }^{3}$, C. Vasconcelos ${ }^{3,4} .{ }^{1}$ Serviço de Medicina, Hospital de São Marcos, Braga, Portugal; ${ }^{2}$ Serviço de Medicina, Porto, Portugal; ${ }^{3}$ Unidade de Imunologia Clínica, Porto, Portugal; ${ }^{4}$ Abel Salazar Biomedical Sciences Institute - University of Porto, UMIB, Porto, Portugal

Background: Cervical cancer is a potentially preventable consequence of Human Papillomavirus (HPV). HPV vaccination is recommended in most countries for all young women, preferentially before sexual activity begins. In Portugal, HPV vaccination is available in either bivalent (genotypes 6,18$)$ or tetravalent $(6$, 11,16 e 18) vaccines. Both have aluminum as an adjuvant, a substance arguably capable of inducing inflammatory adjuvant syndromes. Systemic Lupus Erythematosus (SLE) mostly afflicts women of childbearing age, the very target population for HPV vaccination. There are conflicting reports in the literature regarding both the efficacy and safety of this vaccine in SLE patients. This question is particularly pressing as HPV infection prevalence seems to be increased in SLE patients.

Objectives: To analyze the safety of HPV vaccination in a SLE patient cohort followed at a university hospital.

Methods: Retrospective single-center (35 year long, 436 SLE patient cohort) review of all female SLE patients' local and online national records on HPV vaccination and cervical cancer screening. Data on activity (using SLEDAI-2K scoring) and concomitant drug use were reviewed for the two years before and after vaccination date.

Results: Of the 463 SLE patients, 420 were women (91\%), of which 322 had clinical information about yes/no HPV vaccination. Twenty-seven of the patients $(8 \%)$ had one of HPV vaccines, but only $13(4 \%)$ had full information on activity before and after vaccination. Mean current age was 33,8 years and a minimum of 8,4 years of follow-up after HPV vaccination. Twenty-there $(85 \%)$ were vaccinated with the tetravalent vaccine. Pre-vaccination mean SLEDAI score of was 5.9: due to arthritis $(n=5 ; 38,5 \%)$, low complement 